\title{
MODELACIÓN ESPACIO-TEMPORAL DE LA INCIDENCIA ACUMULADA DE COVID-19 EN MUNICIPIOS DE CHIAPAS
}

\author{
SPATIO-TEMPORAL MODELING OF THE CUMULATIVE INCIDENCE OF \\ COVID-19 IN MUNICIPALITIES OF CHIAPAS
}

Gerardo Núñez Medina gerardo.nm1@gmail.com 
Para citar este artículo:

Núñez Medina, G. (2020). Modelación espacio-temporal de la incidencia acumulada de COVID-19 en municipios de Chiapas. ESPACIO I+D, INNOVACIÓN MÁS DESARROLLO, 9(25). https://doi.org/10.31644/IMASD.25.2020.a01

\section{RESUMEN}

El trabajo tiene como finalidad analizar la evolución de la tasa de incidencia acumulada de COVID-I 9 en los municipios de Chiapas, entre los meses de Febrero a Julio del año 2020, a partir de la aplicación de tres modelos bayesianos jerárquicos espacio-temporales ajustados mediante la Aproximación Anidada Integrada de Laplace (INLA) con el paquete R-INLA que incluyen la estimación de efectos aleatorios estructurados y no estructurados espaciales y temporales. Los resultados muestran la existencia de un efecto de interacción espacio-temporal que permite modelar las variaciones espacio-temporales de las tasas estimadas de incidencia de COVID-I 9 a nivel municipal, las cuales son presentadas en una serie de mapas.

\section{Palabras clave}

Modelos bayesianos, INLA, pandemia, COVID-19, modelos lineales generalizados. 


\section{- Abstract-}

The purpose of the work is analyze the evolution of cumulative incidence rate of COVID-19 in municipalities of Chiapas between months of February to July of 2020 year, based on the application of three hierarchical spatial-temporal bayesian models adjusted by Integrated Nested Laplace Approximation (INLA) method with the R-INLA package that include the estimation of spatial and temporal structured and unstructured random effects. The results indicate the existence of space-time interaction effects that allows modeling the space-time variations of estimated incidence rates of COVID-19 at the municipal level, which are presented in some maps.

\section{Keywords}

Bayesian models, INLA, pandemic, COVID-19, generalized linear models. 
I trabajo hace una revisión de la evolución espacio-temporal de la incidencia de la enfermedad COVID-19 en el Estado de Chiapas, México, a través del uso de modelos bayesianos espacio-temporales con tendencia dinámica no paramétrica. El objetivo es evaluar la velocidad de propagación del virus, medida en términos de la tasa de incidencia acumulada de contagios, a lo largo de los municipios de la entidad y de seis meses de evolución de la pandemia.

La pandemia inicio en Wuhan, China, en noviembre de 2019, propagándose primero a Europa y después al Norte de América. Los primeros casos de COVID-1 9 en México se registraron el mes de febrero de 2020; posteriormente llegaron a Chiapas, donde el primer caso positivo se registró en la ciudad de Tuxtla Gutiérrez, en marzo del mismo año'. Se trató de una estudiante de 18 años, quien regresó de ltalia luego del cierre de la escuela donde estudiaba, debido al incremento de casos positivos de COVID-I 9 en el país europeo.

Con la finalidad de modelar con mayor veracidad la evolución de casos positivos de coronavirus en Chiapas, se utilizó la fecha de aparición de síntomas, reportada por los pacientes confirmados con COVID-19, en lugar de la fecha de ingreso del paciente a la unidad de atención, debido a que puede existir un rezago temporal importante entre la aparición de síntomas y el arribo a la unidad médica.

La pandemia en Chiapas ha evolucionado de forma rápida, durante los meses analizados, alcanzó un total de 9,043 casos confirmados hasta el último día de julio, y se propagó en 98 de los 118 municipios del Estado. Es importante señalar que la mayor incidencia de casos confirmados de coronavirus, ha ocurrido en los municipios con mayor densidad poblacional, como:Tuxtla Gutiérrez, Tapachula, San Cristóbal de las Casas y Comitán, en contraste con los 20 municipios señalados de no presentar ningún contagio, debido, básicamente, a una combinación importante de factores entre los que se destacan las medidas de aislamiento social, los elevados niveles de aislamiento geográfico y las estrategias de prevención, implementadas por los gobiernos Federal, Estatal y Local.

A pesar del incremento señalado en el número de casos confirmados, es importante señalar que las estrategias de prevención de la COVID-19 implementadas, han logrado contener, al menos parcialmente, la evolución

$1 \quad$ El estudio analiza la fecha de inicio de síntomas de COVID-19, los primeros casos reportados en Chiapas datan de inicios del mes de Febrero de 2020, de manera que los casos fueron agregados en periodos de 30 días, incluyen los meses de febrero a julio. 
natural de la epidemia (Ramos, 2020), lo que puede verse con claridad en la gráfica I, donde desde el mes de mayo se observa una reducción continuada en la incidencia de casos de COVID-19 confirmados.

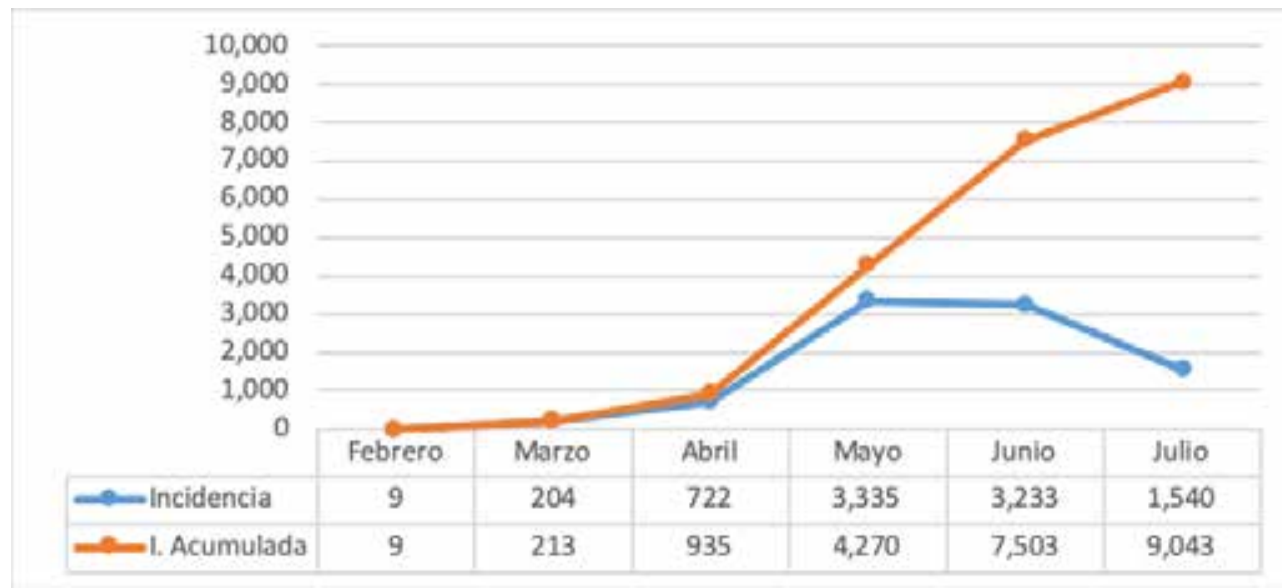

Gráfica 1: Incidencia e Incidencia Acumulada de COVID-19 en Chiapas. Fuente: elaboración propia con datos de la Secretaria de Salud

No obstante, la reducción observada en el número de nuevos contagios de la COVID-19 en la entidad, es importante señalar que, el riesgo de un nuevo brote está presente y que por tanto, se debe continuar con la aplicación de programas de control de la pandemia, y con las medidas orientadas a detectar y atender oportunamente los casos positivos, lo que implica continuar brindando atención médica en toda la entidad, especialmente en los municipios con mayores niveles de vulnerabilidad social y económica.

\section{MATERIALES Y MÉTODO}

Los datos relativos a la evolución espacio-temporal de la COVID- I 9 en los municipios de Chiapas, fueron recopilados de la Base de Datos publicada por la Dirección de Información del Sistema de Vigilancia Epidemiológica de la Secretaría de Salud de México. Los datos abarcan el periodo comprendido entre el I de febrero y el 31 de Julio del 2020. Se procesaron un total de 9,660 casos registrados, utilizando la fecha de inicio de síntomas reportada y asignando la entidad y municipio de residencia del paciente (no el municipio donde se presta la atención). La incidencia de casos positivos de COVID-I9 fueron agrupados y acumulados por mes, con lo que estimaron las tasas de incidencia e incidencia acumulada por municipio. 


\section{Modelos espacio-temporales}

Los datos espacio-temporales pueden definirse como una sucesión indexada, de manera que:

$$
Y(s, t)=\left\{y(s, t) \mid(s, t) \in \mathfrak{R}^{2} \times \mathfrak{R}\right\}
$$

representa un proceso espacio-temporal indexado; donde y(s,t) es el valor observado en el punto $s$ en $n$ áreas espaciales, al tiempo $t$ en $T$ momentos (Blangiardo \& Cameletti, 20I5; p:235), por lo que, es posible representar fenómenos espacio-temporales empleando modelos lineales (generalizados) ${ }^{2}$ con tendencia paramétrica en el componente temporal, al utilizar la función "identidad" como enlace de la variable predictora, definiendo en modelo:

$$
y_{i t}=\eta_{i t}=\beta_{0}+u_{i}+v_{i}+\left(\beta+\delta_{i}\right) t
$$

donde $\beta 0$ representa la intersección o resultado medio esperado, mientras que las funciones ui y vi representan los efectos espacialmente estructurados y espacialmente no estructurados del área i.

El efecto autoregresivo condicional intrínseco ICAR(I) asociado al componente espacial estructurado, es modelado a partir de una distribución condicional dada por:

$$
u_{i} \mid u_{-i} \sim N\left(\frac{1}{\# \mathrm{~N}(i)} \sum_{j=i}^{n} c_{i j} u_{j}, s_{i}{ }^{2}\right)
$$

para toda $\mathrm{i} \neq \mathrm{j}$, donde $\mathrm{c}_{\mathrm{ij}}$ representa el criterio de vecindad; $\mathrm{si} \mathrm{c}_{\mathrm{ij}}=\mathrm{I}$ las áreas i y j son vecinas, en caso contrario $c_{i j}=0$, mientras que la varianza $s_{i}{ }^{2}=\sigma_{u}{ }^{2}$ $\# \mathrm{~N}_{\mathrm{i}}$ del área $\mathrm{i}$, (Bivand et al., 20I5) depende del número de vecinos $\mathrm{N}_{(\mathrm{i})}$.

La especificación Besag - York - Molliè (BYM) de la varianza, utiliza la ecuación anterior para establecer implícitamente que la varianza de regiones con muchos vecinos será menor que la varianza de regiones con pocos vecinos, así, la especificación de BYM supone que el componente espacial no estructurado vi tiene una distribución previa dada por vi $\sim \operatorname{Normal}(0$, $\left.\sigma v^{2}\right)$, donde $\sigma v^{2}$ representa la variabilidad de los efectos aleatorios espaciales no estructurados, mientras que el parámetro $\sigma u^{2}$ controla la variación entre efectos aleatorios espacialmente estructurados (Blangiardo \& Cameletti, 20I5).

2 El caso de los datos espaciales referenciados a un área, a menudo se utilizan modelos lineales de efectos mixtos generalizados, dado que las variables se cuantifican en un número discreto de ubicaciones. 
En la ecuación (I), el parámetro $\beta$ representa el efecto temporal global de todas las áreas, mientras $\delta$ i representa la tendencia diferencial específica del área i al año t, por lo que $\boldsymbol{\delta}$ i se define como un parámetro "previo" intercambiable.

En general, los modelos espacio-temporales suponen la existencia de términos espaciales y temporales separables en el predictor lineal, por lo que la estructura de su matriz de covarianza puede separarse como el producto de: una matriz de efectos espaciales puros y una matriz de efectos temporales puros (Martino \& Rue, 20l0), lo que da origen a cuatro tipos de interacciones que combinan efectos estructurados y no estructurados para las dimensiones espacial y temporal.

\section{MODELOS CON TENDENCIA DINÁMICA NO PARAMÉTRICA}

Los modelos de cohorte paramétrico como el definido por la ecuación (I) imponen restricciones sobre la linealidad del efecto temporal global $(\beta)$ y la tendencia diferencial $\delta i$, en contraste con los modelos no paramétricos quienes permiten modelar tendencias temporales utilizando formulaciones dinámicas a través de: efectos temporales estructurados $\rho t$ y efectos temporales no estructurados $\varphi t$, (Rue \& Martino \& Chopin, 2009) utilizando la identidad como función liga:

$$
y_{s t}=\eta_{s t}=\beta_{0}+u_{i}+v_{i}+\rho_{t}+\varphi_{t}
$$

la tendencia temporal estructurada $\rho t$ se modelada dinámicamente utilizando una caminata aleatoria de orden uno $(\mathrm{rw}(\mathrm{I}))$ definida como:

$$
\rho_{t} \mid \rho_{t-1} \sim N\left(\rho_{t-1}, \sigma^{2}\right) \text {. }
$$

La tendencia temporal no estructurada $\varphi$ t tiene una distribución $\mathrm{N}(0$, $\mathrm{I} / \tau \varphi$ ) (Schrödle \& Held, 20II). Los componentes $\beta 0$, ui y vi se definen como en el modelo (I).

$\mathrm{Si}$, además de modelar de forma dinámica las tendencias espacial y temporal, se busca controlar la interacción espacio-tiempo, es necesario agregar un componente al modelo (2), para lo que se introduce el término yit, con lo que se obtiene la siguiente especificación:

$$
y_{s t}=\eta_{s t}=\beta_{0}+u_{i}+v_{i}+\rho_{t}+\varphi_{t}+\gamma_{i t}
$$

el efecto de interacción espacio-tiempo yit sigue una distribución gaussiana dada por: $\gamma$ it $\sim$ Normal $(0, \mathrm{I} / \tau \gamma)$ (Sharafifi et al, 20l8). Los elementos restantes se definen como en el modelo (2). 
Dado que el modelo (3) asume que la interacción espacio-temporal se presenta a través de un efecto espacial no estructurado vi y una tendencia temporal estructurada $\rho_{t}$, su matriz de covarianza espacio-temporal se escribe como el producto de:

$$
R_{\delta}=R_{v} \otimes R_{\rho}
$$

donde $R v=I$ y $R_{\rho}$ representa la estructura de vecindades especificada a través de la caminata aleatoria de primer orden y $\otimes$ representa el producto Kronecker.

\section{AJUSTE DE MODELOS}

Los modelos espacio-temporales: paramétrico (I), dinámico no paramétrico (2) y no paramétrico de interacción espacio-temporal (3), serán utilizados para ajustar los datos relativos a la incidencia acumulada de la COVID-19, durante los meses de febrero a julio de 2020, para todos los municipios de Chiapas. Los datos comprenden el número de casos de COVID confirmados y referenciados al municipio de residencia, en la fecha en que el enfermo declaró que iniciaron sus síntomas, de manera que la tasa de incidencia acumulada de coronavirus se estima como el cociente del total de casos confirmados de COVID-19, acumulados hasta el mes t, y el número total de casos esperados acumulados hasta el mismo mes, con lo que se tiene:

$$
T I A_{i, t}=\frac{Y_{i, t}}{E_{i, t}}
$$

Lo que representa la tasa de riesgo de enfermar de COVID-19 en el municipio i, al mes t (Ebrahimipour, et al, 2016). Los casos esperados $E_{i, t}$ representan el número total de casos que se esperaría ocurrieran, en el municipio i, al mes t, si la población del municipio presentará el mismo comportamiento que el observado en la población estatal. La forma de calcular el número esperado de casos $E_{i, t}$ es utilizando el método de estandarización indirecta, mismo que supone:

$$
E_{i, t}=r_{t} \times n_{i, t}
$$

donde $r_{t}$ representa la tasa de incidencia acumulada de casos de COVID-1 9 en el Estado de Chiapas, al mes t; esto es, el número total de casos acumulados de COVID-19 dividido por la población total del Estado de Chiapas al mes 
t (Riebler, et al, 2016). Por otra parte, $n_{i, t}$ representa la población total del municipio i al mes $t$.

Una vez estimadas las tasas de incidencia acumulada de COVID-19, para la totalidad de municipios de Chiapas, en los meses señalados, la incidencia acumula de casos de la COVID-19, se ajustará empleando los modelos (I), (2) y (3), presentados en la sección anterior, para ello, se supondrá que los casos acumulados presentan una distribución poisson, es decir la ocurrencia de casos de COVID-19 presenta un comportamiento dado por $Y_{i} \sim$ Poisson $\left(\lambda_{i}\right)$ donde el parámetro $\lambda i$ representa la incidencia media de TIAi expresada como $\lambda_{i}=E_{i} r_{i}$, donde Ei representa el número esperado de casos acumulados de COVID-19 en el municipio $i$.

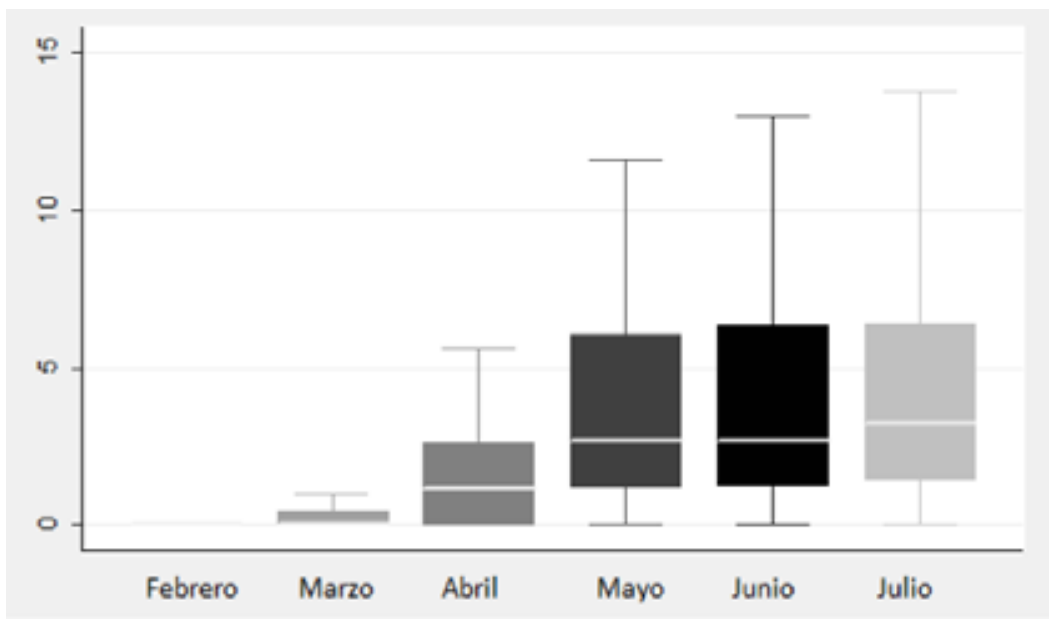

Gráfica 2: Evolución mensual de las tasas de incidencia acumulada de COVID-19 municipales, en Chiapas. Fuente: elaboración propia con datos de la Secretaria de Salud

Los modelos espacio-temporales propuestos, se ajustarán considerando que los efectos espaciales y temporales son separables, además, el efecto espacial estructurado se ajustará usando un modelo ICAR, la tendencia temporal estructurada se modelará utilizando una caminada aleatoria de orden I, mientras que para los efectos no estructurados se supondrá que estos siguen una distribución normal independiente e idénticamente distribuida. En el caso de la estimación de parámetros e hiperparámetros se utilizarán especificaciones previa vagas con el fin de evitar un sobre ajuste de los modelos.

La estimación de parámetros de los modelos propuestos fue hecha utilizando el paquete Integrated Nested Laplace Approximation (INLA) desarrollado por (Rue et al 2009) e implementado en lenguaje de programación $\mathrm{R}$. Este paquete permite hacer inferencias bayesianas rápidas y eficientes en modelos estructurales aditivos (que forman parte de un conjunto más amplio de modelos Bayesianos jerárquicos) (Martins et al, 
20I3). El enfoque INLA es una alternativa menos costosa computacionalmente que el método tradicional MCMC.

Los resultados obtenidos de las estimaciones posteriores de los parámetros para los modelos (I), (2) y (3) que incluyen la estimación de: efectos fijos, se presentan en la tabla I; efectos aleatorios se presentan en la tabla 2 y el mapa I, e incluyen los efectos temporales estructurados y no estructurados presentados en la gráfica 2; y distribuciones posteriores de los hiperparámetros presentados en la gráfica 3.

\section{Calidad de ajuste de los modelos}

Al momento se han propuesto tres modelos espacio-temporales: modelo paramétrico clásico (I), modelo dinámico no paramétrico (2) y modelo no paramétrico de interacción espacio-temporal(3). Una vez estimados los modelos (I) a (3) la forma de identificar el modelo es través de criterio de información como el Akaike (AIC) o el Criterio de información de Desviación (DIC) donde valores más bajos son indicadores de un mejor ajuste, y una diferencia DIC de 3-5 se considera significativa. (Lawson, 20I5). El modelo con mejor ajuste debe tener un AIC bajo y un número efectivo pequeño de parámetros.

El modelo seleccionado será aquel que alcance el criterio de información de Akaike (AIC) más bajo, en términos de:

$$
\mathrm{AIC}=-2 \mathrm{l}(\hat{\theta})+2 \mathrm{k}
$$

donde $\mathrm{I}\left(\theta^{\wedge}\right)$ es la función de máxima de log-verosimilitud y $\mathrm{k}$ es el número de parámetros del modelo (Blangiardo \& Cameletti, 20 I5). El criterio DIC permite confirmar los resultados obtenidos.

\section{Tabla I}

Criterios de información Akaike y DIC de los modelos (I) a (3)

\begin{tabular}{cccc}
\hline & Modelo & AIC & DIC \\
(1) & $y_{i t}=\eta_{i t}=\beta_{0}+u_{i}+v_{i}+\left(\boldsymbol{\beta}+\boldsymbol{\delta}_{i}\right) t$ & 3050.29 & 3073.25 \\
(2) & $y_{s t}=\eta_{s t}=\beta_{0}+u_{i}+v_{i}+\rho_{t}+\varphi_{t}$ & 3109.20 & 3127.76 \\
(3) $y_{s t}=\eta_{s t}=\beta_{0}+u_{i}+v_{i}+\rho_{t}+\varphi_{t}+\gamma_{i t}$ & 3008.03 & 3030.60 \\
\hline
\end{tabular}

Fuente: elaboración propia

La tabla I presenta los criterios de información AIC y DIC para los modelos (I) a (3); en la misma se muestra que el modelo (3) que incluye un término de interacción espacio-temporal, cuya distribución previa es una caminata 
aleatoria de orden I, presentó un AIC sensiblemente más bajo que los modelos restantes. De esta forma se utilizará el modelo no paramétrico de interacción espacio-temporal para modelar el comportamiento de las tasas de incidencia acumulada del virus SARS COVID-19 en el estado de Chiapas.

\section{RESULTADOS}

Los valores de las estimaciones posteriores de los efectos fijos para la media, desviación estándar y cuántiles realizadas a los modelos (I) a (3), se presentan en la tabla I. El parámetro $\beta 0$ representa el logaritmo natural del nivel medio de la tasa de incidencia acumulada de COVID-19 para el estado de Chiapas, durante el periodo analizado, lo que equivale a un riesgo de $\exp (-0.048)=0.953$ para el caso el modelo no paramétrico de interacción espacio-temporal. Se debe señalar que la tasa de incidencia acumulada promedio observada se estimó en 3.032, como puede verse en la gráfica 2.

\section{Tabla 2}

Estimaciones posteriores de efectos fijos de los modelos espacio-temporales

\begin{tabular}{cccccc}
\hline Modelo paramétrico & mean & sd & Q1 & Q2 & Q3 \\
$\beta 0$ & -1.312 & 0.095 & -1.501 & -1.311 & -1.127 \\
year & 0.012 & 0.009 & -0.005 & 0.012 & 0.029 \\
Modelo no paramétrico & mean & sd & Q1 & Q2 & Q3 \\
$\beta 0$ & -1.227 & 0.099 & -1.423 & -1.227 & -1.034 \\
Modelo de interacción & mean & sd & Q1 & Q2 & Q3 \\
$\beta 0$ & -0.048 & 2590.538 & -5086.148 & -0.121 & 5081.806 \\
\hline
\end{tabular}

Fuente: elaboración propia con base en estimaciones de R-INLA

La tabla 3 muestra las estimaciones posteriores de la media, desviación estándar y cuartiles de los efectos aleatorios de los modelos espaciotemporales ( 1 ) a (3). En el caso del modelo no paramétrico de interacción espacio-temporal, la media de la densidad posterior del efecto espacial estructurado ui, es más pequeña que la presentada por el efecto espacial no estructurado vi, lo que muestra la importancia relativa del efecto de los contagios entre municipios. 
Tabla 3

Estimaciones posteriores de efectos aleatorios de los modelos espacio-temporales

\begin{tabular}{lcccccc}
\hline & Modelo paramétrico & mean & sd & Q1 & Q2 & Q3 \\
ui & Efecto espacial estructurado & 1.711 & 0.428 & 0.992 & 1.673 & 2.664 \\
vi & Efecto espacial no estructurado & 1.458 & 0.785 & 0.539 & 1.262 & 3.501 \\
$\beta$ & Efecto temporal global & 669.726 & 322.029 & 267.901 & 596.127 & 1496.186 \\
& Modelo no paramétrico & mean & sd & Q1 & Q2 & Q3 \\
ui & Efecto espacial estructurado & 0.936 & 0.131 & 0.695 & 0.931 & 1.210 \\
vi & Efecto espacial no estructurado & 1955.511 & 1973.912 & 131.504 & 1368.654 & 7199.003 \\
pt & Efecto temporal estructurado & 30865.678 & 26630.544 & 4520.841 & 23530.318 & 101036.083 \\
$\varphi t$ & Efecto temporal no estructurado & 30109.846 & 25511.926 & 4577.809 & 23135.855 & 97364.132 \\
Modelo no paramétrico de interacción & mean & sd & Q1 & Q2 & Q3 \\
ui & Efecto espacial estructurado & 2089.183 & 546.525 & 1280.541 & 1993.961 & 3553.927 \\
vi & Efecto espacial no estructurado & 2305.892 & 388.167 & 1606.390 & 2267.574 & 3294.092 \\
$\rho t$ & Efecto temporal estructurado & 14278.740 & 3164.152 & 8948.889 & 13880.661 & 22673.242 \\
$\varphi t$ & Efecto temporal no estructurado & 16083.408 & 3695.816 & 9314.816 & 15712.501 & 25587.534 \\
yit & Efecto int. espacio-temporal & 97.368 & 7.460 & 83.523 & 96.909 & 115.192 \\
\hline
\end{tabular}

Fuente: elaboración propia con base en estimaciones de R-INLA

El valor medio estimado para los efectos temporales estructurados y no estructurados del modelo de interacción espacio-temporal, presentados en la tabla 3, muestra que las estimaciones de los efectos temporales son mucho mayores que las alcanzadas por los efectos espaciales (estructurados y no estructurados), lo que hace evidente la mayor relevancia del tiempo (en relación con el espacio) como un factor explicativo fundamental para modelar la evolución de los contagios por el virus COVID-I 9.

La variación observada entre los efectos temporales estructurados $\rho t$ y los efectos temporales no estructurados $\varphi t$, donde la media de la densidad posterior de la precisión de los efectos estructurados es relativamente menor, que la estimada para los efectos temporales no estructurados implica que la capacidad explicativa del modelo de interacción espacio-temporal debe tomar en cuenta la interacción de los efectos espaciales y temporales (Schrödle \& Held, 20I I). Esto se debe a que las variaciones entre efectos espaciales y entre efectos temporales son relativamente pequeñas, comparadas con la variación entre efectos espaciales y temporales (ver tabla 3).

La media de la densidad posterior estimada para el término de interacción espacio-temporal yit resulta ser pequeña en relación con las medias estimadas para los efectos temporal y espacial (Martins et al., 20I2), sin embargo, logra capturar adecuadamente la dependencia espacial y la evolución temporal de las tasas de incidencia acumuladas de COVID-19. 


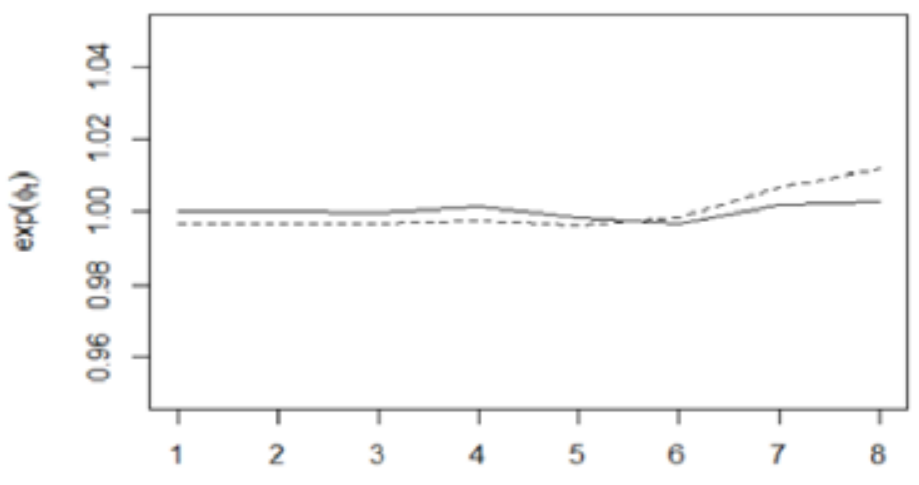

Efecto temporal no estructurado línea solida $\varphi$ t.

Efectos temporal estructurados línea punteada $\rho t$.

Gráfica 3. Tendencia posterior de los efectos temporales estructurados $\rho$ t y no estructurados $\varphi t$ del modelo de interacción espacio-temporal Fuente: elaboración propia con base en estimaciones de R-INLA

La Gráfica 3 presenta la tendencia posterior de los efectos temporales estructurados $\rho t$ y no estructurados $\varphi t$ del modelo de interacción espaciotemporal (modelo (3)), en la misma se observa que los efectos temporales estructurados inician con valores por abajo de uno, no obstante, a partir del mes de junio el peso del efecto estructural comienza a crecer de forma sostenida, con lo que se puede esperar que, en los próximos meses, el efecto de las variaciones aleatorias temporales tengan una menor incidencia sobre la evolución de los contagios por la COVID-19 en el estado de Chiapas.

La Gráfica 4 muestra las distribuciones posteriores de los efectos aleatorios de los componentes espaciales y temporales, estructurados y no estructurados, además del efecto de interacción espacio-temporal del modelo (3). La estimación de tales distribuciones permite estimar con gran precisión la probabilidad posterior de observar tasa de incidencia acumulada de COVID- 19 mayores a uno.

Las tasas acumuladas de incidencia de COVID-19, observadas durante los meses de Marzo, Mayo y Julio del año 2020 para los municipios de Chiapas, pueden observarse en el mapa I, lado izquierdo, las mismas, muestran la ausencia de contagios en un importante número de municipios durante el mes de Marzo. En los mismos mapas puede verse la evolución espacial y temporal del número de municipios que presentan contagios, así como el surgimiento durante los meses de Mayo a Julio de un conglomerado de municipios con tasa de contagio por debajo del promedio Estatal (municipios en color azul). 



Efectos espaciales estructurados ui Efectos espaciales no estructurados vi Efectos temporales estructurados $\rho t$

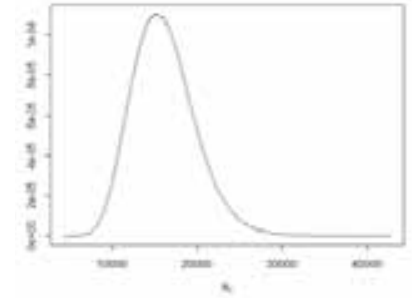

Efectos temporales no estructurados $\varphi t$

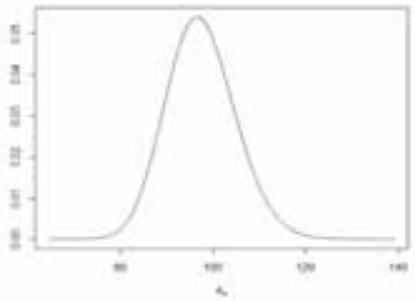

Efectos de interacción espacio-temporal yit

Gráfica 4: Distribuciones posteriores de efectos aleatorios espaciales y temporales. Fuente: elaboración propia con base en estimaciones de R-INLA

La parte derecha del mapa I, presenta información sobre la media posterior estimada, del modelo de interacción espacio-temporal, para la tasa de incidencia acumulada de COVID-I 9 en los municipios Chiapas para los meses de Marzo, Mayo y Julio. Las tasas se presentan en escala logarítmica, para evitar un problema de sobre dispersión de los datos. En general, se puede ver que el modelo ajusta adecuadamente el comportamiento observado de las tasas y completa (interpola) el valor medio de las tasas de COVID-I 9 en los municipios donde no se cuenta con información. Es importante señalar que los municipios con mayores tasas de incidencia acumulada de COVID- 19 son:Tuxtla Gutiérrez, Tapachula y Reforma. 


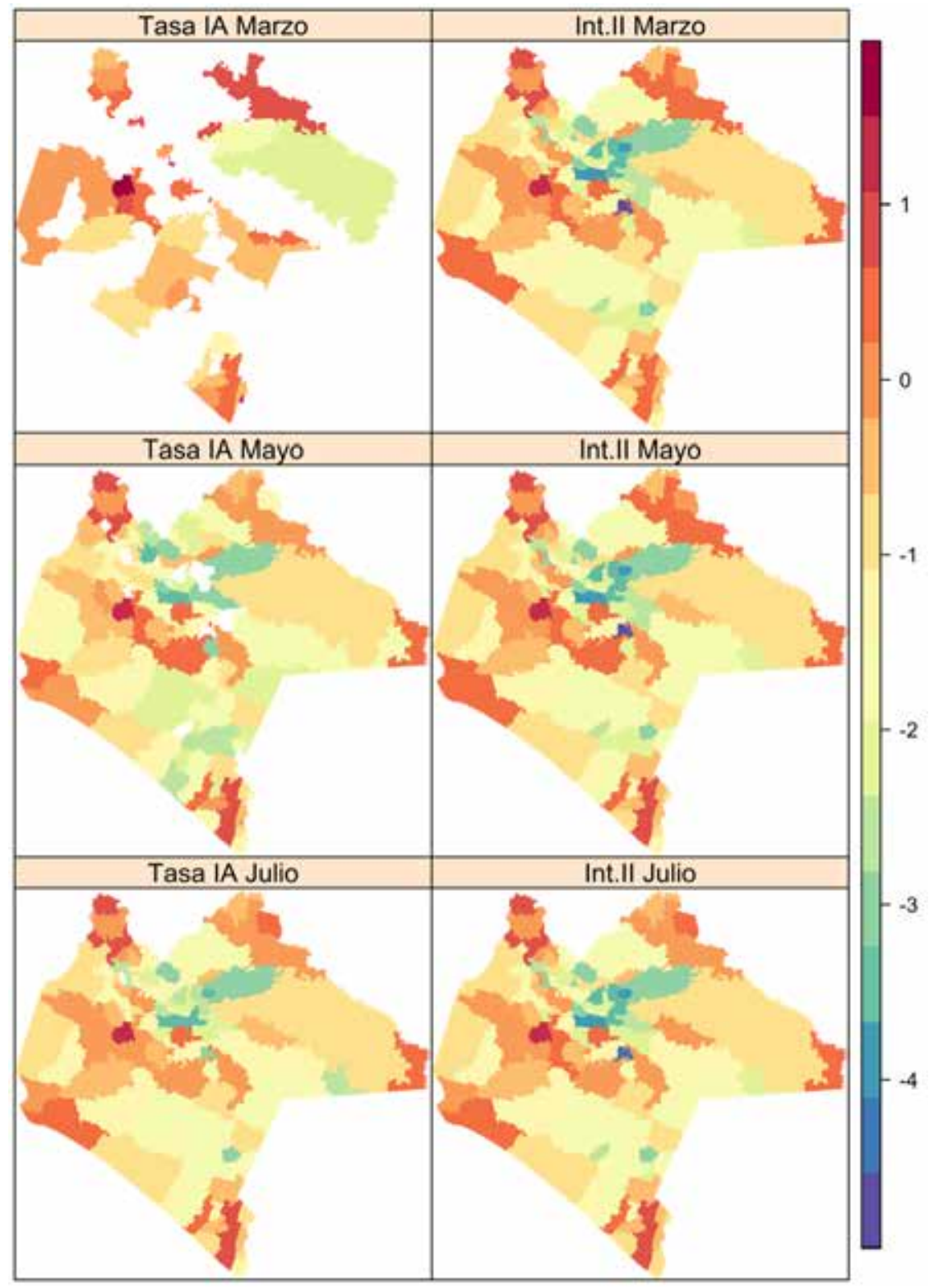

Mapa 1. Tasa de incidencia acumulada de COVID-19, para Chiapas y Medias posteriores de riesgo relativo estimado por el modelo (3). Fuente: elaboración propia con base estimaciones de R-INLA

\section{CONCLUSIONES}

A lo largo de este trabajo se han descrito y aplicado tres modelos espaciotemporales bayesianos jerárquicos ajustados con el paquete R-INLA con el objetivo de analizar la evolución de la tasa de incidencia acumulada de COVID-I9 en los municipios de Chiapas, entre los meses de Febrero a Julio. Es decir, se trata de un primer ejercicio de estimación que incorpora la 
interacción de efectos aleatorios espacio-temporales con el fin de estimar las variaciones mensuales a nivel municipal de la tasa de incidencia de COVID-19.

Las técnicas de modelado espacio-temporal bayesiano existentes en R-INLA pudieron aplicarse exitosamente a la evolución de las tasas de incidencia acumulada de COVID-19, dada la disponibilidad y oportunidad de datos, lo que permitió analizar las variaciones geográficas y temporales, además, dado que los modelos estudiados presentaron efectos aditivos, con un único efecto espacio-temporal, fue posible reducir el costo de cómputo asociado a la estimación.

Como puede observarse en el mapa I, el municipio de Tuxtla Gutiérrez es el epicentro de la pandemia en Chiapas, lidera significativamente los casos diarios de infecciones por COVID-19, sin embargo, es seguido por los municipios de Tapachula y Reforma, quienes registran un importante riesgo de contagio asociado. Los resultados presentados señalan que, en general, no son los municipios con mayor densidad poblacional quienes enfrentan las mayores tasas de contagio, sino que la evolución espacio-temporal de la pandemia ha afectado de manera diferencial a los municipios de la entidad, protegiendo a aquellos que tienen mayores niveles de aislamiento geográfico y/o social.

Si bien, enfrentar la pandemia implica desde luego incrementar las capacidades del sistema de salud del Estado, es importante mencionar que la prevención y contención de los contagios puede resultar en una mejor estrategia, cuya orientación debe incluir el detectar y aislar los casos observados, buscando con ello interrumpir el ciclo de transmisión del virus. A pesar de que los resultados indican una reducción paulatina y constante, la tasa de incidencia de COVID-I9 (ver gráfica I), el número de municipios donde el riesgo de contagio es bajo ha disminuido también de forma sostenida durante los meses de estudio, lo que implica que pese a que se registran menos contagios, existe una mayor dispersión espacial de COVID- I 9 (ver mapa I) sobre el territorio chiapaneco. 


\section{REFERENCIAS}

Blangiardo, M.; Cameletti, M. (2015) Spatial and Spatio-Temporal Bayesian Models with R-INLA. John Wiley \& Sons: Chichester, West Sussex, UK.

Bivand, Roger \& Gómez Rubio, Virgilio \& Rue, Håvard. (2015). Spatial Data Analysis with R-INLA with Some Extensions. Journal of statistical software. 63.1-31.

Martino, S., Rue, H. (2010). Implementing Approximate Bayesian Inference using Integrated Nested Laplace Approximation: a manual for the inla program. Disponible en http://citeseerx.ist.psu.edu/viewdoc/download ;jsessionid=0740AA9C1C62B6CF7A148F17015204B3?doi=10.1.1.142.3 845\&rep=rep $1 \&$ type $=p d f$

Martins, G., Simpson, D., Lindgren, F., Rue, H. (2012). Bayesian computation with INLA: new features. Norwegian University of Science and Technology Report.

Ebrahimipour, M.; Budke, C.M.; Najjari, M.; Cassini, R.; Asmarian, N. (2016). Bayesian spatial analysis of the surgical incidence rate of human cystic echinococcosis in north-eastern Iran. Acta Trop. 163, 80-86.

Ramos C. (2020). Covid-19: la nueva enfermedad causada por un coronavirus. Salud Pública de México. 2020;62:225-227. https://doi.org/10.21149/11276

Riebler, A.; Sørbye, S.H.; Simpson, D.; Rue, H. (2016). An intuitive Bayesian spatial model for disease mapping that accounts for scaling. Stat. Methods Med. Res. 25, 1145-1165.

Rue, H.; Martino, S.; Chopin, N. (2009) Approximate Bayesian inference for latent Gaussian models by using integrated nested Laplace approximations. J. R. Stat. Soc. Ser. B (Stat. Methodol.), 71, 319-392.

Schrödle, B., Held L. (2011). Spatio-temporal disease mapping using INLA. Environmetrics 22 (6), 725-734.

Sharafifi, Z.; Asmarian, N.; Hoorang, S.; Mousavi, A. (2018). Bayesian spatiotemporal analysis of stomach cancer incidence in Iran, 2003-2010. Stoch. Environ. Res. Risk Assess. 32, 2943-2950. 